\title{
Abstraction and Reality. Dealing with ...
}

\author{
Jana Moser $^{\text {a, }}$, Christian Hanewinkel ${ }^{\text {a }}$ \\ ${ }^{a}$ Leibniz Institute for Regional Geography,j_moser@leibniz-ifl.de, c_hanewinkel@leibniz-ifl.de \\ * Corresponding author
}

Keywords: Generalization, Basic Geometries, Thematic Cartography, Size and Area Changes

\begin{abstract}
:
Publicly accessible geometries at small scales form a good basis for visualising spatial data. But how do colleagues beyond cartography deal with the information they contain, be they geographers, archaeologists or biologists? What errors arise when calculations are carried out with generalised data and how can the transparency of geometries be increased so that it becomes visible which operations are possible with them and which are not?
\end{abstract}

In Germany, the Federal Agency for Cartography and Geodesy (BKG) offers geometries for administrative units and hydrography, among others, in the scale range between 1:250,000 and 1:2.5 million. The basis for the geometries generalised in the respective scales is the idea of the Official Topographic Cartographic Information System (Amtliches Topographisch-Kartographisches Informationssystems/ATKIS). This results in the Digital Landscape Model (DLM) and, for example, the Administrative Regions (VG), which are updated annually.

The Leibniz Institute for Regional Geography (IfL) has used BKG data as a starting point for further generalisations. The aim was to generate Germany-wide geometries for thematic maps at small scales, which

- $\quad$ can vary between poster size and desktop monitor

- $\quad$ allow visualisation at the municipality level in all scales

- $\quad$ can be used for both printed and digital products

- $\quad$ have a high graphic quality and

- $\quad$ have the smallest possible data volume for digital and mobile products.

In a first review and assessment of the BKG geometries it became clear that they were still too fragmented and detailed for IfL purposes in the sense of thematic maps. Therefore, both the administrative boundaries and the hydrography were further simplified as basic elements for thematic maps in the IfL. The processing was carried out in three generalisation levels, starting from the BKG 1:1 million scale: developing the scales 1:1 million, 1:2.5 million and 1:5 million. This is based on the experience of the National Atlas of the Federal Republic of Germany.

In the course of the process, it became clear that significant changes in the length of line segments were recorded at all scales. For example, the length of all lines at the municipality level was reduced by more than $15 \%$. In terms of area, the greatest changes occurred at the scale of 1:1 million. For example, the area of the federal states decreased by about 327 $\mathrm{km}^{2}$, which corresponds to the area of the city of Dresden or about 3 times the area of Florence. Larger changes in area size due to the generalisation process also occurred in the municipalities when, for example, enclaves or exclaves were dissolved by falling below the minimum dimension.

The presentation will illustrate such and other changes in the generalisation process using the example of the administrative geometries of the Federal Republic of Germany. Possibilities of making the use of such data transparent and making it available for open access will be discussed. This should contribute to the realisation that the geometric data in such information systems are not suitable for the calculation of indicators. 